\title{
Influência das condições de cura na reologia dos novos depósitos de argilas bentoníticas da Paraíba
}

\section{(Influence of cure conditions in the rheology of the new bentonitic clay deposits of Paraíba)}

\author{
I. C. G. Morais ${ }^{1}$, I. A. Silva ${ }^{1}$, B. M. A. B. Buriti ${ }^{1}$, R. R. Menezes ${ }^{1}$, G. A. Neves ${ }^{1}$, H. C. Ferreira \\ ${ }^{1}$ Universidade Federal de Campina Grande, Unidade Acadêmica de Engenharia de Materiais, \\ 58429-900, Campina Grande, PB, Brasil
}

\begin{abstract}
Resumo
Novos depósitos de argilas esmectíticas foram descobertos no estado da Paraíba, Brasil, melhorando a questão da escassez das argilas de melhor qualidade. Estudos mostram que o teor de umidade, tempo de cura e capacidade de troca de cátions de bentonitas aditivadas com carbonato de sódio influenciam diretamente na reologia de dispersões em meio aquoso. Sendo assim, este trabalho objetivou analisar a influência da variação de umidade e tempo de cura nas trocas catiônicas de argilas bentoníticas para fluidos de perfuração aquosos. As argilas foram beneficiadas por hidrociclonagem e caracterizadas física, química e mineralogicamente. Em seguida, foram aditivadas com carbonato de sódio variando o teor de umidade e o tempo de cura. A capacidade de troca de cátions foi determinada antes e após o processo de aditivação. As propriedades reológicas e de filtração foram determinadas por meio de viscosidades aparente e plástica e o volume de filtrado. Os resultados permitiram concluir que o tempo de cura e teor de umidade tem influência na capacidade de troca catiônica e nas propriedades reológicas e de filtração. Após 15 dias de cura das argilas, houve decréscimo das capacidades de troca de cátions e das propriedades reológicas e de filtração.

Palavras-chave: argilas bentoníticas, umidade, tempo de cura, capacidade de troca de cátions, fluidos de perfuração.
\end{abstract}

\begin{abstract}
New deposits of smectic clays were discovered in the state of Paraíba, Brazil, improving the issue of the scarcity of better quality clays. Studies have shown that the moisture content, curing time and cation exchange capacity of bentonites added with sodium carbonate directly influence the rheology of dispersions in aqueous media. Thus, this work aimed to analyze the influence of moisture variation and curing time on the cation exchange of bentonite clays for aqueous drilling fluids. The clays were benefited by hydrocyclone and were characterized physically, chemically and mineralogically. Then, they were added with sodium carbonate varying the moisture content and the curing time. The cation exchange capacity was determined before and after the additivation process. The rheological and filtration properties were determined by apparent and plastic viscosities and the volume of filtrate. The results allowed to conclude that curing time and moisture content had an influence on cation exchange capacity and rheological and filtration properties. After 15 days of cure of the clays, there was a decrease in cation exchange capacity and rheological and filtration properties.
\end{abstract}

Keywords: bentonite clays, moisture, time to cure, cation exchange capacity, drilling fluids.

\section{INTRODUÇÃO}

Argilas bentoníticas são formadas pela desvitrificação e subsequente alteração química de material vítreo, oriundo da decomposição de rochas vulcânicas, de preferência ácidas, depositadas em lagos ou rios de baixa turbulência, cujo principal argilomineral de sua composição é a montmorilonita. Este tipo de argila é formado por lamelas compostas por uma folha octaédrica de alumina $\left(\mathrm{Al}_{2} \mathrm{O}_{3}\right)$ entre duas folhas tetraédricas de sílica $\left(\mathrm{SiO}_{2}\right)$, ligadas entre si por átomos de oxigênio [1-4]. Devido à extração desordenada das argilas bentoníticas no estado da Paraíba, Brasil, as argilas de melhores composições mineralógicas se exauriram

*brunamichelebrito@gmail.com
[5]. As variedades comercializadas atualmente apresentam um elevado grau de contaminação por minerais acessórios, como quartzo, feldspato, gipsita, albita, halita, calcita, pirita, carbonatos de sódio e outros, que por vez comprometem suas aplicações tecnológicas [6]. Alguns estudos com objetivo de eliminar esses minerais acessórios têm sido desenvolvidos nos últimos anos com destaque o trabalho [7], que utilizou a técnica da hidrociclonagem [7-10].

A eliminação de minerais acessórios de argilas bentoníticas é uma etapa de elevada importância, pois resulta nas melhoraria das propriedades específicas, como granulometrias finas,elevadacargasuperficial,altacapacidade de troca de cátions contendo cerca de 80 a $150 \mathrm{meq} / 100 \mathrm{~g}$, sendo superior aos demais argilominerais que geralmente não ultrapassa $40 \mathrm{meq} / 100 \mathrm{~g}$, elevada área específica, 
propriedades de intercalação de outros componentes entre as camadas, inchamento quando em presença de água e resistência à temperatura e aos solventes $[11,12]$. Em sua estrutura cristalográfica, as argilas bentoníticas possuem o alumínio substituído parcial ou totalmente por íons, principalmente $\mathrm{Mg}^{2+}$ ou $\mathrm{Fe}^{3+}$ [2-4]. As propriedades físicoquímicas dos argilominerais estão intimamente associadas aos fenômenos de superfície e carga superficial. Dentre esses fenômenos tem-se a capacidade de troca de cátions, caráter anfótero e acidez superficial. Nas cargas resultantes das substituições isomórficas, o desequilíbrio da dissociação de grupos $\mathrm{OH}^{-}$atrai, especialmente, 'cátions trocáveis' de diferentes cargas e tamanhos, sendo adsorvidos nos argilominerais, modificando assim o comportamento a nível tecnológico e industrial $[5,12,13]$.

A maior parte das aplicações industriais e acadêmicas de argilas é feita usando-se bentonitas sódicas. As bentonitas brasileiras, cálcicas, podem passar por um processo de ativação usando carbonato de sódio (barrilha) para a troca dos cátions interlamelares de $\mathrm{Ca}^{2+}$ por $\mathrm{Na}^{+}$. $\mathrm{O}$ íon $\mathrm{Na}^{+}$hidrata-se mais que o $\mathrm{Ca}^{2+}$. Além disso, a distância interlamelar nas bentonitas é maior quando as lamelas têm suas cargas compensadas pelo íon sódio, de menor valência, permitindo a penetração de uma maior quantidade de água no espaço entre as lamelas. Isso explica porque a capacidade de expansão da bentonita sódica é muito maior do que a do tipo cálcica [13]. O armazenamento e/ ou a qualidade da aditivação depende da umidade da argila e da dosagem do sódio no processo de aditivação, onde a umidade da argila deve ser considerada e controlada $[6,14$, 15]. Esse armazenamento pode apresentar maior ou menor grau de estabilidade em suas propriedades reológicas com o tempo, devido às suas características floculadas-gel serem susceptíveis ao efeito do armazenamento [6, 16]. Fatores como a umidade, a fração argilosa e diferentes processos industriais de aditivação também podem ser capazes de modificar as propriedades reológicas das argilas [6], sendo extremamente necessário para a indústria da área conhecer qual tempo de cura e teor de umidade ótimos para o processo. Esse trabalho tem como objetivo analisar a influência das condições de cura na reologia dos novos depósitos de argilas bentoníticas da Paraíba.

\section{MATERIAIS E METODOLOGIA}

Para esse estudo foi selecionada uma argila bentonítica, referida como AM1, fornecida pela Mina Fazenda Campos, Olivedos, pertencente aos novos jazimentos da Paraíba, Brasil. A amostra foi beneficiada pela técnica de hidrociclonagem utilizando um hidrociclone (RWK 42L, Netzch AWK, Alemanha, capacidade de separação de partículas com diâmetro de 2 a $5 \mu \mathrm{m}$ ), com a finalidade de eliminar parte dos minerais acessórios que compromete seu uso em fluido de perfuração. Esse ensaio foi realizado com uma configuração de $4 \mathrm{~mm}$ para o ápex e $5 \mathrm{~mm}$ para o vórtex, utilizando um volume de $30 \mathrm{~L}$ de água destilada para uma massa de 1,368 kg de argila. Após beneficiamento, a amostra foi seca ao sol, onde diariamente foram retiradas alíquotas e realizadas as medições de umidade, visando seu controle, e em seguida passada em peneira ABNT n ${ }^{\circ} 200$ $(0,074 \mathrm{~mm})$. $\mathrm{O}$ aditivo utilizado no processo de aditivação foi o carbonato de sódio PA $\left(\mathrm{Na}_{2} \mathrm{CO}_{3}\right.$, pureza de $99,5 \%$, Labsynth, Diadema, SP).

A caracterização física, química e mineralógica da amostra beneficiada foi realizada pelos seguintes métodos: análise granulométrica por difração a laser (AG, Cillas 1064); difração de raios X (DRX, Shimadzu XRD 6000), com radiação $\mathrm{K} \alpha$ do $\mathrm{Cu}(40 \mathrm{kV} / 30 \mathrm{~mA})$ e velocidade do goniômetro de $2 \% / \mathrm{min}$ e passo de $0,02^{\circ}$, com faixa de varredura de $5^{\circ}$ a $40^{\circ}$. Foi utilizado o etileno glicol para a confirmação da amostra como argila bentonítica. A análise termogravimétrica (TG, Shimadzu DTG $60 \mathrm{H}$ ) e análise térmica diferencial (DTA, BP RB-3000) foram realizadas com taxa de aquecimento de $12,5{ }^{\circ} \mathrm{C} / \mathrm{min}$, temperatura máxima para ambos de $1000^{\circ} \mathrm{C}$ e o padrão utilizado na DTA foi o óxido de alumínio $\left(\mathrm{Al}_{2} \mathrm{O}_{3}\right)$ calcinado a $1200{ }^{\circ} \mathrm{C}$.

As umidades foram controladas na temperatura ambiente (forma de secagem de melhor controle [6]) nas variações de $25 \%, 35 \%, 45 \%, 50 \%$ e $60 \%$, em seguida aditivadas com o $\mathrm{Na}_{2} \mathrm{CO}_{3}$ na proporção de $125 \mathrm{meq} / 100 \mathrm{~g}$ de argila seca (esta concentração foi escolhida por ter conduzido os melhores resultados em [17]), levando-se em consideração a umidade, sendo calculados o equivalente grama e o milequivalente grama do carbonato envolvido, tomando por base a capacidade de troca de cátions (CTC, parâmetro para aditivação) da amostra original (policatiônica). Pela massa da amostra que foi utilizada, foi calculada a massa de carbonato de sódio necessária para aditivar a argila [6]. $\mathrm{Na}$ aditivação as argilas foram submetidas a intervalos de 7 , 15, 30 e 45 dias de cura, para realização do estudo reológico e de filtração. As dispersões bentoníticas, policatiônicas e monocatiônicas, foram preparadas com a concentração especificada na norma da Petrobras [18], sendo de $24,3 \mathrm{~g} / 500 \mathrm{~mL}$ de água deionizada (4,86\% em massa); em seguida foram homogeneizadas com agitador Marconi $500 \mathrm{~W}$ a aproximadamente $150000 \mathrm{rpm}$ por $20 \mathrm{~min}$, logo após permanecerem em repouso por $24 \mathrm{~h}$. Os parâmetros reológicos, viscosidades aparente (VA) e plástica (VP), foram medidos por meio de viscosímetro Fann 35A segundo as equações:

$$
\mathrm{VA}=300 \frac{\theta}{\mathrm{N}}
$$

onde, VAé a viscosidade aparente dada em mPa.s, N (mPa.s) é a velocidade de rotação em 600 rpm do viscosímetro Fann e $\theta$ é a deflexão medida no viscosímetro a esta velocidade, e:

$$
\mathrm{VP}=300 \frac{\theta_{2}-\theta_{1}}{\mathrm{~N}_{2}-\mathrm{N}_{1}}
$$

onde, VP é a viscosidade plástica dada em mPa.s, $\theta_{2}$ e $\mathrm{N}_{2}$ com leitura a $600 \mathrm{rpm}$ e $\theta_{1}$ e $\mathrm{N}_{1}$ com leitura a $300 \mathrm{rpm}$. O volume de filtrado (VF) foi determinado por meio de ensaio realizado em filtro prensa, no qual foi inserida a dispersão 
e recolhido o filtrado durante o período de $30 \mathrm{~min}$ após a aplicação de uma pressão de $690 \pm 35 \mathrm{kPa}(100 \pm 5$ psi).

\section{RESULTADOS E DISCUSSÃO}

$\mathrm{Na}$ Tabela I estão contidos os valores de análise química por fluorescência de raios X (EDX, Shimadzu 720). Analisando os resultados, verificou-se que a amostra apresentou elevados teores de $\mathrm{SiO}_{2}$ e $\mathrm{Al}_{2} \mathrm{O}_{3}$, por serem os principais componentes dos minerais de argilas. Segundo $[19,20]$, baixo teor de sílica $\left(\mathrm{SiO}_{2}\right)$ e alto teor de alumina $\left(\mathrm{Al}_{2} \mathrm{O}_{3}\right)$ são indicativos do alto teor de minerais de argila presente; o oposto disso, elevado teor de sílica e baixo teor de alumina, é indicativo da baixa proporção de minerais de argila e grandes quantidades de quartzo livre na amostra. Para o teor de $\mathrm{Fe}_{2} \mathrm{O}_{3}$ observou-se que a amostra apresentou teor superior a $8 \%$, típico das argilas do Município de Boa Vista, PB [6, 15]. A remoção dos óxidos de Fe promove diminuição na CTC de argilominerais [21]. Os óxidos alcalino-terrosos $\mathrm{CaO}$ e $\mathrm{MgO}$ apresentaram teores inferiores a $1 \%$, podendo ser uma indicação da presença de carbonatos $[19,20]$. O valor de $\mathrm{TiO}_{2}$ foi relativamente baixo, inferior a $1 \%$. Comparando os valores determinados, verificou-se que as amostras de argilas estudadas possuíram composição química típica das argilas esmectíticas do estado da Paraíba.

Tabela I - Composição química (\% em massa) da amostra AM1 obtida pela fluorescência de raios X.

[Table I - Chemical composition (wt\%) of AMI sample obtained by X-ray fluorescence.]

\begin{tabular}{cccccccc}
\hline $\mathrm{SiO}_{2}$ & $\mathrm{Al}_{2} \mathrm{O}_{3}$ & $\mathrm{MgO}$ & $\mathrm{Fe}_{2} \mathrm{O}_{3}$ & $\mathrm{CaO}$ & $\mathrm{TiO}_{2}$ & $\begin{array}{c}\text { Outros } \\
\text { oxidos }\end{array}$ & $\mathrm{PR}$ \\
\hline 54,53 & 21,03 & 2,57 & 9,75 & 0,73 & 0,93 & 0,43 & 9,5 \\
\hline
\end{tabular}

PR - perda ao rubro.

A Fig. 1 ilustra a curva de DRX da amostra AM1 com e sem etileno glicol. Observou-se que a amostra estudada apresentou a seguinte composição mineralógica: argilomineral esmectítico, montmorilonita (JCPDS 291497), caracterizado pelas distâncias interplanares basais

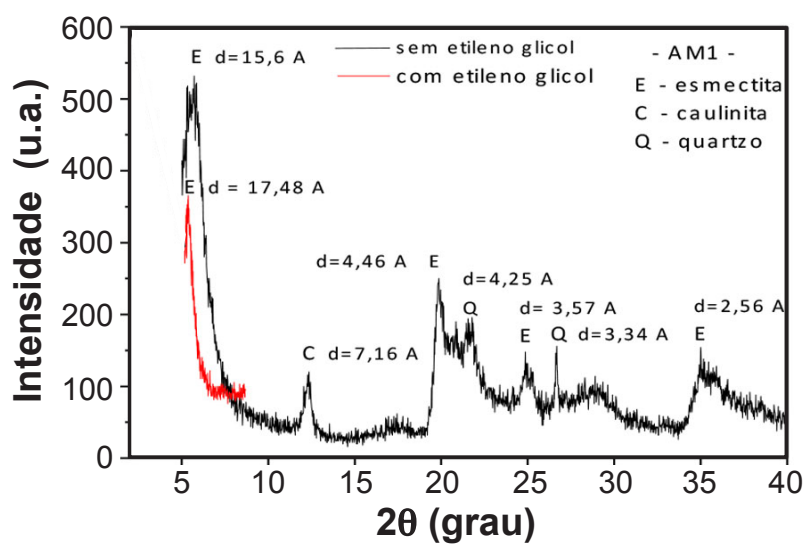

Figura 1: Difratogramas de raios X para a amostra AM1. [Figure 1: X-ray diffraction patterns for sample AM1.] de $15,6,4,46,3,57$ e $2,56 \AA$, que foi confirmado pelo uso do etileno glicol que aumentou a distância interplanar de 15,6 para 17,48 ̊̊; argilomineral caulinítico (JCPDS 140164) caracterizado pela distância interplanar basal de 7,16 ̊; mineral acessório, como quartzo (JCPDS 461045), caracterizado pelas distâncias interplanares basais de 4,25 e 3,34 Å. No geral, os espectros da difração de raios $\mathrm{X}$ confirmaram, qualitativamente, que as argilas apresentaram curvas típicas das argilas bentoníticas com outros minerais acessórios, sendo similares aos estudos realizados em $[6,7,22-26]$.
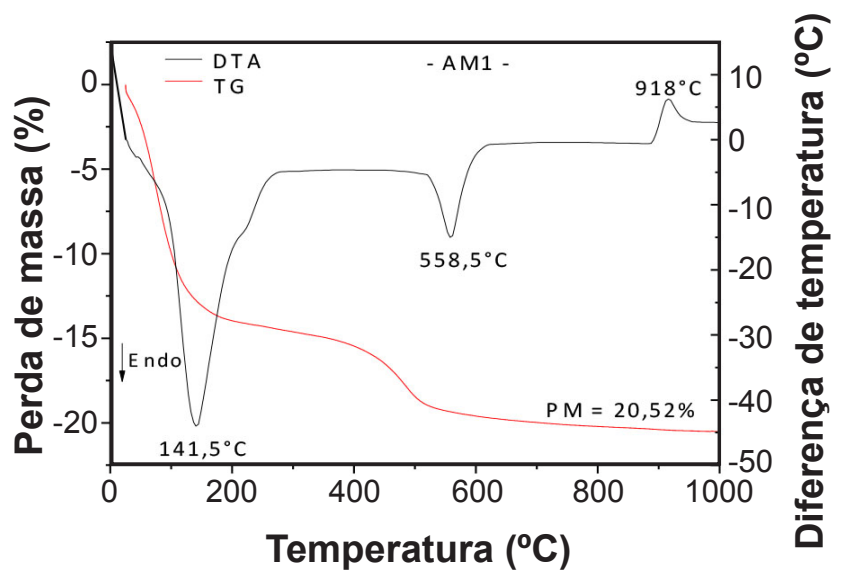

Figura 2: Curvas termogravimétrica e de análise térmica diferencial da amostra AM1, em taxa de aquecimento de $12,5^{\circ} \mathrm{C} / \mathrm{min}$.

[Figure 2: Thermogravimetric and differential thermal analysis curves of sample AM1, under heating rate of $12.5^{\circ} \mathrm{C} / \mathrm{min}$.]

A Fig. 2 ilustra as curvas de DTA e TG da amostra AM1. Verificou-se que a amostra estudada apresentou perda de massa total de aproximadamente $20,52 \%$, relacionada a perdas de água (livre, adsorvida e coordenada), matéria orgânica, hidroxilas e carbonatos. A partir da análise térmica diferencial observaram-se as seguintes transformações térmicas: pico endotérmico em aproximadamente $141,5{ }^{\circ} \mathrm{C}$ relacionado à presença de água livre e absorvida; pico endotérmico em aproximadamente $558,5{ }^{\circ} \mathrm{C}$ relacionado à presença de hidroxila da esmectita e caulinita; pico exotérmico em aproximadamente $916{ }^{\circ} \mathrm{C}$ relacionado à nucleação da mulita. Os resultados obtidos corroboraram os apresentados em [5-7, 17, 24, 26, 27] e verificou-se que argila apresentou comportamento térmico similar às argilas bentoníticas do estado da Paraíba.

A Fig. 3 ilustra a curva de distribuição de tamanho de partículas da amostra estudada. A amostra AM1 apresentou comportamento bimodal observando-se uma maior concentração de partículas entre 0,07 e $9 \mu \mathrm{m}$ e ausência total ou parcial da fração com tamanho de partícula $>10 \mu \mathrm{m}$. Verificou-se um elevado percentual de volume acumulado com diâmetro inferior a $2 \mu \mathrm{m}$, no valor de 83,33\%, provavelmente relativo à concentração de fração argilosa resultante do processo de beneficiamento. A amostra AM1 apresentou diâmetro em $50 \%$ em volume cumulativo (D50) de 0,53 $\mu \mathrm{m}$ e diâmetro médio $(\mathrm{DM})$ de $1,09 \mu \mathrm{m}$. Esses 
resultados conduzem a melhores propriedades reológicas, pois, conforme [19, 20], melhores propriedades reológicas são encontradas em amostras com menores diâmetros médios e com maiores teores de partículas com $\mathrm{x}<2 \mu \mathrm{m}$ ). Os resultados obtidos corroboraram os apresentados em [6, 27].

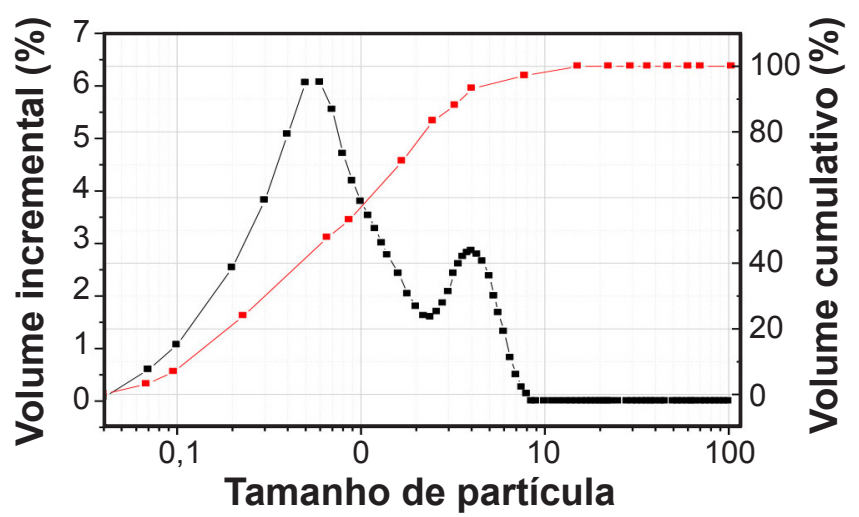

Figura 3: Curvas de distribuição granulométrica da amostra AM1. [Figure 3: Granulometric distribution curves of sample AM1.]

A Tabela II apresenta os resultados reológicos (VA, VP), de filtração (VF) e a CTC da amostra beneficiada, antes da aditivação, com base na norma da Petrobras [18]. Foi possível observar que as propriedades reológicas e de filtração da amostra beneficiada não satisfazem os requisitos estabelecidos pela norma [18], apresentando resultados abaixo dos especificados, com exceção para o VF. Embora a fração argilosa presente na amostra seja elevada (Fig. 3) e percentuais de minerais acessórios tenham sido retirados com o processo de beneficiamento, a presença das argilas na forma cálcica e policatiônica conduz a resultados reológicos insatisfatórios [6], uma vez que as argilas quando sódicas são mais fortemente hidratadas, apresentando inchamento mais significativo das partículas, causando assim um grande aumento em suas propriedades reológicas [6].

A Fig. 4 corresponde aos resultados reológicos CTC (a), VA (b), VP (c) e de filtração VF (d) da amostra aditivada a $25,35,45,50$ e $60 \%$ de umidade nos tempos de cura de 7, 15,30 e 45 dias com base na norma da Petrobras [18]. Analisando as Figs. 4a a 4c pode-se observar que a amostra atingiu as especificações estabelecidas [18] para todos os teores de umidade e tempos de cura, onde a norma [18] determina valor de VA maior ou igual a 15,0 mPa.s, valor de VP maior ou igual a 4,0 mPa.s e valor de VF menor ou igual a $18,0 \mathrm{~mL}$. Observando os resultados de CTC (Fig. 4d) verificam-se valores entre 42 e $94 \mathrm{meq} / 100 \mathrm{~g}$. No intervalo de 7 a 15 dias de cura houve um crescimento da CTC, obtida com o aumento dos teores de umidade, sendo os melhores resultados alcançados no tempo de cura de 15 dias. Após esse tempo de cura, verificou-se que as amostras começaram a apresentar decaimento progressivo nos valores de CTC, que também foi observado em [6, 15]. Com relação a VA (Fig. 4a), foi possível observar que o tempo de cura ideal foi de 15 dias para a maioria dos teores de umidade (concentrações), com exceção da amostra de $35 \%$ de umidade que conduziu um tempo de cura ideal de 30 dias. Para a VP (Fig. 4b), o tempo de cura ideal para a maioria das concentrações foi de 30 dias, com exceção do teor de umidade de $60 \%$, que conduziu um tempo de cura ideal de 15 dias. Com relação aos resultados de VF (Fig. 4c), observou-se que o tempo de cura ideal para todos os teores de umidade foi de 30 dias, reduzindo os valores de VF ao longo dos dias, evidenciando a provável diferenciação entre a água estrutural e a água adicionada durante o processo. No geral, observou-se influência dos teores de umidade (concentração do $\mathrm{Na}_{2} \mathrm{CO}_{3}$ ) e tempo de cura na CTC da amostra, havendo consequentemente influência também nas propriedades reológicas das dispersões obtidas, uma vez que os íons permutáveis influenciam fortemente nas propriedades físico-químicas e reológicas das argilas bentoníticas e de suas dispersões.

Segundo estudos realizados em [6], as argilas bentoníticas podem apresentar maior ou menor grau de estabilidade em suas propriedades reológicas, sendo aquelas com características floculadas-gel as mais susceptíveis ao efeito do tempo de cura. De forma semelhante, o aumento do teor de umidade durante o envelhecimento tende a melhorar a cinética da reação entre $\mathrm{o} \mathrm{Na}_{2} \mathrm{CO}_{3}$ e as argilas, resultando em aumento nos valores de VAe VPe diminuição do VF, o que é reforçado pelo tempo de cura e aumento da umidade [6, 28-30], fato este observado também no presente estudo. Zandonadi [31] avaliou o comportamento reológico de bentonitas nacionais como agente tixotrópico de fluidos de perfuração base água. Foram propostos 18 diferentes ensaios de troca por sódio das bentonitas policatiônicas, sendo escolhido o processo 18 como o mais eficiente. Posteriormente foram realizadas pesquisas sistemáticas de troca de cátions incluindo tratamentos térmicos de forma a melhorar os coeficientes de difusão diminuindo o tempo de cura e melhorando sua eficiência [32]. Estudos [29, 30] confirmam o efeito benéfico dos elevados teores de umidade na cinética da reação de troca que ocorre entre o $\mathrm{Na}_{2} \mathrm{CO}_{3}$ nas esmectitas antes de aditivadas. Porém, o que se observou nesse estudo é que existe um limite de teor de umidade ideal e tempo de cura, que quando ultrapassado os resultados que sucedem passam a ser insatisfatórios. Os resultados

Tabela II - VA, VP, VF e CTC da amostra.

[Table II - VA, VP, VF and CEC of the sample.]

\begin{tabular}{cccccc}
\hline Amostra & VA (mPa.s) & VP (mPa.s) & VF $(\mathrm{mL})$ & CTC (meq/100 g) & Umidade (\%) \\
\hline AM1 & 11,0 & 3,0 & 17,0 & 72 & $10 \%$ \\
Valor aceitável [18] & $\geq 15,0$ & $\geq 4,0$ & $\leq 18,0$ & - & - \\
\hline
\end{tabular}



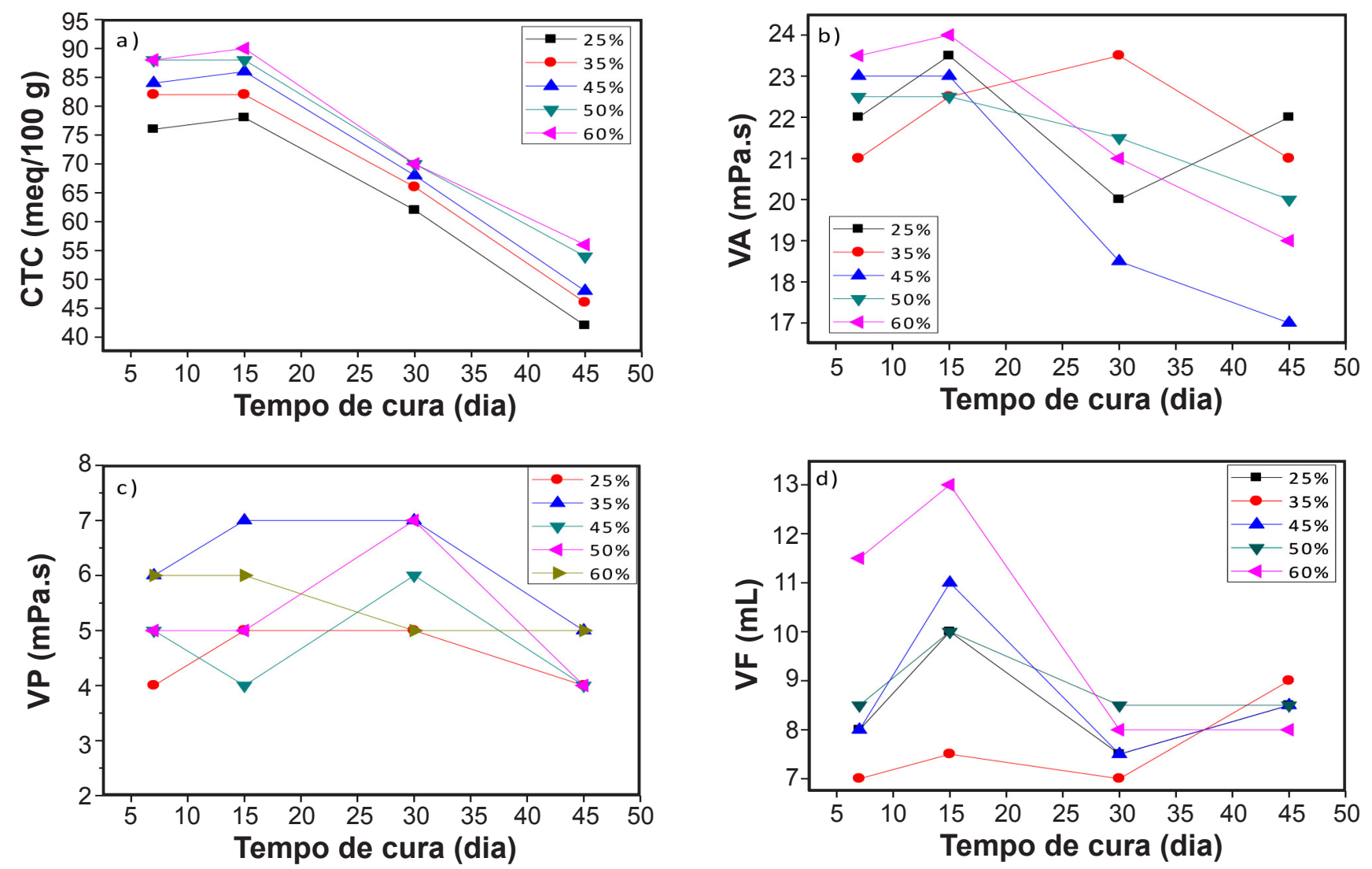

Figura 4: Resultados de CTC (a), VA (b), VP (c), e VF (d) da amostra aditivada a 25, 35, 45, 50 e 60\% de umidade nos tempos de cura de $7,15,30$ e 45 dias.

[Figure 4: Results of $C E C(a), A V(b), P V(c)$, and $F V(d)$ of the sample added at 25, 35, 45, 50 and 60\% humidity at curing times of 7, 15, 30 and 45 days.]

reológicos inferiores com o decorrer do tempo de cura devemse provavelmente ao fato de haver uma reversibilidade das reações de troca de cátions, consequentemente a perda das propriedades frente aos mais diversos aspectos, fornecendo ainda dados importantes sobre a vida útil ou validade desses insumos industriais; dessa forma, a reversibilidade das reações pode ser verificada de forma indireta pelo controle reológico [6].

\section{CONCLUSÕES}

Com base nesse estudo, pôde-se concluir que houve influência direta das variações de umidade e tempo de cura na capacidade de troca de cátions (CTC) de argilas bentoníticas aditivadas com $\mathrm{Na}_{2} \mathrm{CO}_{3}$, consequentemente nas propriedades reológicas e de filtração, sendo o ponto ótimo para teor de umidade de $60 \%$ e 15 dias de cura. Concluiu-se também que esses parâmetros são de fundamental importância em relação às suas aplicações industriais, pois tempo de cura elevado, ou seja, superior ao ponto ótimo, começa a afetar a CTC e as propriedades reológicas e de filtração.

\section{REFERÊNCIAS}

[1] R. Barbosa, D.D.S. Morais, E.M. Araújo, T.J.A. Mélo,
Cerâmica 58, 347 (2012) 363.

[2] M.I. Abdou, A.M. Al-Sabagh, M.M. Dardir, Egypt. J. Pet. 22 (2013) 53.

[3] P. Souza Santos, Tecnologia de argilas, vol. 1, Edgard Blücher, S. Paulo (1992) 350.

[4] F.R. Valenzuela Díaz, P. Souza Santos, H.L. Souza Santos, Quím. Ind. 42, 7 (1992) 33.

[5] R.R. Menezes, L.F.A. Campos, H.S. Ferreira, L.N. Marques, G.A. Neves, H.C. Ferreira, Cerâmica 55, 336 (2009) 349.

[6] I.A. Silva, F.K.A. Sousa, H.S. Ferreira, H.S. Ferreira, G.A. Neves, H.C. Ferreira, Cerâmica 63, 365 (2017) 109.

[7] A.J.A. Gama, J.M.R. Figuerêdo, J.M. Cartaxo, M.A.Gama, G.A. Neves, H.C. Ferreira, Cerâmica 63, 367 (2017) 336.

[8] J.M.R. Costa, I.A. Silva, H.S. Ferreira, R.R. Menezes, G.A. Neves, H.C. Ferreira, Cerâmica 58, 348 (2012) 419.

[9] J.M.R. Figueirêdo, J.P. Araújo, I.A. Silva, J.M. Cartaxo, G.A. Neves, H.C. Ferreira, Mater. Sci. Forum 798-799 (2015) 21.

[10] V.C. Marques, H.C. Silva, A.M.T. Rodrigues, J.M. Cartaxo, R.R. Menezes, H.S. Ferreira, Cerâmica 61, 359 (2015) 285.

[11] I.A. Silva, F.K.A. Sousa, R.R. Menezes, H.S. Ferreira, G.A. Neves, H.C. Ferreira, Cerâmica 64, 369 (2018) 109. 
[12] I.A. Silva, J.M.R. Costa, R.R. Menezes, H.S. Ferreira, G.A. Neves, H.C. Ferreira, Rev. Esc. Minas 66 (2013) 485. [13] R.R. Menezes, L.R.L. Melo, F.A.S. Fonseca, H.S. Ferreira, G.A. Neves, Rev. Eletr. Mater. Proces. 3 (2008) 36.

[14] H.S. Ferreira, L.F.A. Campos, R.R. Menezes, J.M. Cartaxo, L.N.L. Santana, G.A. Neves, H.C. Ferreira, Cerâmica 59, 350 (2013) 277.

[15] C. Liang, W. Sun, T. Wang, X. Liu, Z. Tong, Colloids Surf. A Physicochem. Eng. Asp. 490 (2016) 300.

[16] J.M.R. Costa, I.A. Silva, H.S. Ferreira, R.R. Menezes, G.A. Neves, H.C. Ferreira, Cerâmica 58, 348 (2012) 419.

[17] P.M. Bastos, B.M.A. Brito, A.J.A. Gama, J.M. Cartaxo, G.A. Neves, L.F.A. Campos, Cerâmica 63, 366 (2017) 187.

[18] Petrobras, "Ensaio de viscosificante para fluidos base água na exploração e produção de petróleo", EP-1EP00011-A (2011).

[19] R. Gaidzinski, J. Duailibi Fh., L.M. Tavares, Appl. Clay Sci. 54 (2011) 47.

[20] R. Gaidzinski, P. Osterreicher-Cunha, J. Duailibi Fh., L.M. Tavares, Appl. Clay Sci. 43 (2009) 98.

[21] G. Kahr, F.T. Madsen, Appl. Clay Sci. 9 (1995) 327.

[22] R.R. Menezes, LN. Marques, L.F.A. Campos, H.C. Ferreira, L. Santana, G.A. Neves, Appl. Clay Sci. 49 (2010) 13.
[23] I.A. Silva, J.M.R. Costa, H.S. Ferreira, R.R. Menezes, G.A. Neves, H.C. Ferreira, Cerâmica 58, 347 (2012) 317.

[24] B.M.A. Brito, J.M. Cartaxo, N.F.C. Nascimento, H.C. Ferreira, G.A. Neves, R.R. Menezes, Cerâmica 62, 361 (2016) 45.

[25] C.I.R. Oliveira, M.C.G. Rocha, A.L.N. Silva, L.C. Bertolino, Cerâmica 62, 363 (2016) 272.

[26] W.P. Gonçalves, V.J. Silva, R.R. Menezes, G.A. Neves, H.L. Lira, L.N. Santana, Appl. Clay Sci. 137 (2017) 259.

[27] I.D.S. Pereira, I.A. Silva, J.M. Cartaxo, R.R. Menezes, L.N.L. Santana, G.A. Neves, H.C. Ferreira, Cerâmica 60, 354 (2014) 223.

[28] C. Karagüzel, T. Çetinel, F. Boylu, K. Çinku, M. Çelik, Appl. Clay Sci. 48 (2010) 398.

[29] R. Shu, W. Sun, X. Liu, Z. Tong, J. Colloid Interface Sci. 444 (2015) 132.

[30] W.Z. Chang, Y.K. Leong, Rheologica Acta 53 (2014) 109.

[31] A.R. Zandonadi, "Estudo tecnológico de argilas montmoriloníticas brasileiras", Tese Dout., USP, São Paulo (1972).

[32] N.F.C. Nascimento, H.C. Ferreira, G.A. Neves, Rev. Eletr. Mater. Proces. 10, 3 (2015) 144.

(Rec. 19/12/2017, Rev. 19/02/2018, 19/03/2018, Ac. 29/03/2018) 